\title{
Invasive Lung Mucinous Adenocarcinoma
}

National Cancer Institute

\section{Source}

National Cancer Institute. Invasive Lung Mucinous Adenocarcinoma. NCI Thesaurus.

Code C136709.

An invasive adenocarcinoma that arises from the lung. It is characterized by the presence

of tall columnar cells and mucin production. This category refers to cases formerly classified as mucinous bronchioloalveolar carcinoma, excluding cases that meet the criteria for adenocarcinoma in situ or mucinous minimally invasive adenocarcinoma. 